\title{
COVID-19 Burden on HIV Patients Attending Antiretroviral Therapy in Addis Ababa, Ethiopia: A Multicenter Cross-Sectional Study
}

Dagmawi Chilot ( $\sim$ dagimchilot21@gmail.com )

University of Gondar College of Medicine and Health Sciences

Yimtubezinash Woldeamanuel

Addis Ababa University

Tsegahun Manyazewal

Addis Ababa University

\section{Research}

Keywords: Coronavirus disease 2019 (COVID-19), Severe acute respiratory syndrome coronavirus 2 (SARS-CoV-2), HIV, clinical care, treatment, antiretroviral therapy, Ethiopia

Posted Date: July 27th, 2021

DOl: https://doi.org/10.21203/rs.3.rs-699963/v1

License: (c) (1) This work is licensed under a Creative Commons Attribution 4.0 International License.

Read Full License

Version of Record: A version of this preprint was published at Frontiers in Medicine on March 2nd, 2022.

See the published version at https://doi.org/10.3389/fmed.2022.741862. 


\section{Abstract \\ Background}

There has been promising progress towards screening, testing, and retaining HIV patients in care in Ethiopia. Concern exists that possible disruptions in HIV programs due to COVID-19 could result in more HIV-related mortality and new HIV infections. This study aimed to investigate the real-time burden of COVID-19 on HIV patients attending antiretroviral therapy.

\section{Methods}

We conducted a facility-based, multicentre, cross-sectional study among HIV patients attending antiretroviral therapy in 10 healthcare facilities in Addis Ababa, Ethiopia, in the COVID-19 pandemic period. Data was collected using adapted, interviewer-based questionnaires, and entered into Epi Info version 7 and exported to SPSS version 26 for analysis.

\section{Result}

A total of 212 patients with HIV were included. Participants who missed visits for refill were 58 (27.4\%). When the effects of other independent variables on appointments/visits for refill were controlled, the following characteristics were found to be the most important pridictors of missed appointments $(P<$ 0.05): age $\geq 55$ [AOR $=6.73,95 \% \mathrm{Cl}(1.495-30.310)]$, fear of COVID-19 [AOR $=24.93,95 \% \mathrm{Cl}(2.798-$ 222.279)], transport disruption [AOR $=4.90,95 \% \mathrm{Cl}(1.031-23.174)]$, reduced income for traveling to health facility $[A O R=5.64,95 \% \mathrm{Cl}(1.234-25.812)]$, and limited access to mask [AOR $=7.67,95 \% \mathrm{Cl}$ (1.303-45.174)], sanitizer [AOR $=0.07,95 \% \mathrm{Cl}(0.007-0.729)$ ] and non-medical support [AOR $=2.32,95 \%$ $\mathrm{Cl}(1.547-12.596)]$. The participants were well aware of the COVID-19 preventive measures. The most costly COVID-19 preventive measures that cause financial burden to the patients were costs for buying facemasks (63.7\%), disinfectants (55.2) and sops for handwashing (22.2). Participants who missed follow-up diagnostic tests were $56(26.4 \%)$. Variables which were found to be statistically significant include the following: age $\geq 55$ [AOR $=0.22,95 \% \mathrm{Cl}(0.076-0.621)]$, partial lockdown [AOR $=0.10,95 \% \mathrm{Cl}$ (0.011-0.833)], limited access to health services [AOR $=0.15,95 \% \mathrm{Cl}(0.045-0.475)$ ], reduced income for traveling to health facility [AOR $=0.18,95 \% \mathrm{Cl}(0.039-0.784)$ ], and unable to get mask [AOR $=0.12,95 \% \mathrm{Cl}$ (0.026-0.543)]. Participants who missed counseling services were 55 (25.9\%). In multivariate logistic regression the following were statisticaly significant: age $\geq 55$ [AOR $=0.21,95 \% \mathrm{Cl}(0.078-0.570)$ ], fear of COVID-19 [AOR $=0.11,95 \% \mathrm{Cl}(0.013-0.912)]$, reduced income [AOR $=0.17,95 \% \mathrm{Cl}(0.041-0.699)]$, unable to get face mask $[A O R=0.19,95 \% \mathrm{Cl}(0.039-0.959)]$, and partial lockdown $[A O R=0.08,95 \% \mathrm{Cl}(0.008-$ 0.790)].

\section{Conclusions}


COVID-19 had a significant burden on HIV patients to attend their routine clinical care and treatment, which may lead to treatment failure and drug resistance. The impact was on their appointments for medication refills and clinical and laboratory follow-ups. Targeted initiatives are needed to sustain HIV clinical care and treatment services and improve the wellbeing of people living with HIV.

\section{Background}

Coronavirus disease 2019 (COVID-19) could be the most catastrophic pandemic in modern history. It has infected over $173,674,509$ people globally and resulted in more than $3,744,408$ deaths as of 09 June 2021 [1]. Countries have been taking strong preventive measures to reduce and curve the transmission [2-4]. Many health care professionals shifted and health facilities were repurposed into targeted COVID19 centers to manage patients [5-7]. Evidence showed these measures have led to restrictions of health facilities to the management of emergency medical conditions and chronic diseases care and treatment services [8, 9]. In Ethiopia, COVID-19 imposed a burden on physical infrastructure and exacerbated the preexisting weaknesses of health systems. As the country has limited numbers of hospitals and health centers, it presented a significant challenge to manage the pandemic and other diseases simultaneously [10-13].

By the end of 2020, it was estimated that 37.6 million people have Human Immunodeficiency Virus (HIV) infection globally and 1.5 million are newly infected. Only 27.4 million of them are on treatment with antiretroviral therapy (ART), which means 10.2 million (27\%) people are still to come [14]. HIV remains highly prevalent in Africa, accounting for more than $67 \%$ of the people living with HIV/AIDS (PLWH) worldwide [15]. Concern exists that possible disruptions in HIV programs due to COVID-19 could result in more HIV-related mortality and new HIV infections.

The double burden of COVID-19 and HIV is one of the major health challenges especially in developing countries with high HIV prevalence [16-18]. PLWH might be particularly at high risk for infection with poor clinical outcomes [19-21]. Containment measures, disruptions to supply chains, and loss of income have the potential to exacerbate the impacts of the pandemic on HIV patients [22]. While these impacts will vary significantly across countries, some recommended providing ART for 3-6 months and others began to offer home delivery services through volunteers to reduce adverse health outcomes $[23,24]$. the extensive demand for physicians has led to the rescheduling of routine HIV patients reviews and hospital visits [25-27]. Fear of COVID-19 exacerbated food insecurity and COVID-19 protective behaviors hindered voluntary HIV testing and healthcare services.

Many countries warned that they are at risk of stock-outs of antiretroviral (ARV) medicines and some have critically low stocks as a result of the pandemic [28]. In addition, PLWH were doubtful regarding the availability of ART services and regarding which HIV clinic to attend in the pandemic period [29].

There are limited real-time patient-level researches regarding how effective and useful country-level COVID-19 interventions were for HIV patients. As well, the impact of the COVID-19 pandemic on HIV at a population level is not well known. With the limited level of evidence in the world and as to our knowledge, 
no research was done regarding the impact of the pandemic on patients attending HIV care and treatment services in Ethiopia. There is an urgent need for adequately powered studies that investigate the impact of COVID-19 on HIV clinical care and treatment to augment the health of people living with HIV.

Thus, this study aimed to investigate the real-time burden of COVID-19 on people living with HIV who were attending ntiretroviral therapy facilities in Addis Ababa, Ethiopia.

\section{Methods}

\section{Design and setting}

A multi-center study was carried out at ten primary health care centres in Addis Ababa, from April 15 to March 30, 2021. The city has 10 sub-cities and 116 woredas and has different government health facilities including six hospitals and 106 public health centers. In Ethiopia, the COVID-19 pandemic is higher in the capital Addis Ababa [30]. Addis Ababa is the highest in HIV prevalence next to Gambella regional state [31]. The study was conducted in 10 health facilities, one in each sub-city which has high HIV patient flow.

\section{Participants}

In this study, the source population was all HIV patients of age $>18$ years attending care and treatment in the selected health centers. The study population were those who were attending care and treatment services during the data collection period. Participants were included if they were I) patients with HIV, as confirmed within the study facilities or result referred from another health facility; II) man or woman aged $\geq 18$ years; III) volunteer to participate in the study. All eligible participants who have been attending clinical care and treatment in those study sites during the data collection period were considered using a predetermined sampling procedure (Table 1). 
Table 1

Sampling procedure

\begin{tabular}{|lll|}
\hline Name of health facility & Sub-city & No of. HIV cases on ART \\
\hline Addis raey HC & Addis ketema & 20 \\
\hline Akaki HC & Akaki kality & 50 \\
\hline Kebena HC & Arada & 18 \\
\hline Goro HC & Bole & 11 \\
\hline Addisu gebya HC & Gulele & 16 \\
\hline Kazanchis HC & Kirkos & 30 \\
\hline Alem bank & Kolfe & 10 \\
\hline T/haymanot HC & Lideta & 36 \\
\hline Woreda 02 HC & Nifas-silk lafto & 10 \\
\hline Woreda 13 HC & Yeka & 11 \\
\hline
\end{tabular}

\section{Data collection}

Adapted, pre-tested and structured questionnaire used to collect primary data for the assessment of the overall impact of COVID-19. The data collection instrument was developed in English and was translated to Amharic, and later back-translated to English to check for any inconsistencies or distortion in the meaning and concepts of the words by another person. Eligible participants who were attending the selected health centers were invited to participate. Participants were given information about the study through an information sheet and signed a consent form if they agreed to be part of the study. The data collectors and supervisors were trained before the actual data collection period regarding the approach, objective of the study, and ethical issues. The data collection was interviewer-administered and the questionnaire includes sections such as sociodemographic characteristics, awareness about preventive measures, care and treatment services.

\section{Data analysis and interpretation}

All questionnaires were checked for completeness every day by the principal investigator and supervisors. Data cleaning was conducted at the end of the data entry. The analysis was done using bivariate and multivariate logistic regression to observe the effects of independent variables on the outcome variable while simultaneously controlling for other potential confounding factors. The raw data entered into Epi Info version 7 to control entry errors and exported to SPSS 26 for analysis.

\section{Results}




\section{Socio-Demographic Characteristics}

A total of $212 \mathrm{HIV}$ patients were enrolled in the study, with a response rate of $100 \%$, and $133(62.7 \%)$ were female. Of the total, $103(48.6 \%)$ were in the age group 35-54 years. Most of them (41.5\%) were married and $59(27.8 \%)$ had attended primary education. One hundred and forty-six $(68.9 \%)$ were Orthodox Christian, and $24.1 \%$ were governmental employees (Table 2 ). 
Table 2

Sociodemographic characteristics of respondents, Addis Ababa, Ethiopia, May 2021.

\begin{tabular}{|c|c|c|c|}
\hline \multicolumn{2}{|l|}{ Variables Category } & \multirow{2}{*}{$\begin{array}{l}\text { Frequency } \\
79\end{array}$} & \multirow{2}{*}{$\begin{array}{l}\text { Percentage } \\
37.3 \%\end{array}$} \\
\hline Sex & Male & & \\
\hline & Female & 133 & $62.7 \%$ \\
\hline \multirow[t]{3}{*}{ Age } & $18-34$ & 55 & $25.9 \%$ \\
\hline & $35-54$ & 103 & $48.6 \%$ \\
\hline & $\geq 55$ & 54 & $25.5 \%$ \\
\hline \multirow[t]{5}{*}{ Marital status } & Single & 50 & $23.6 \%$ \\
\hline & Married & 88 & $41.5 \%$ \\
\hline & Widowed & 40 & $18.9 \%$ \\
\hline & Divorced & 26 & $12.3 \%$ \\
\hline & Separated & 8 & $3.8 \%$ \\
\hline \multirow[t]{5}{*}{ Level of education } & No education & 46 & $21.7 \%$ \\
\hline & Can read and write & 30 & $14.2 \%$ \\
\hline & Primary education & 59 & $27.8 \%$ \\
\hline & Secondary education & 44 & $20.8 \%$ \\
\hline & Diploma and above & 33 & $15.6 \%$ \\
\hline \multirow[t]{5}{*}{ Religion } & Orthodox & 146 & $68.9 \%$ \\
\hline & Muslim & 36 & $17.0 \%$ \\
\hline & Protestant & 21 & $9.9 \%$ \\
\hline & Catholic & 3 & $1.4 \%$ \\
\hline & Others & 6 & $2.8 \%$ \\
\hline \multirow[t]{6}{*}{ Occupation } & Student & 3 & $1.4 \%$ \\
\hline & Daily laborer & 41 & $19.3 \%$ \\
\hline & Merchant & 22 & $10.4 \%$ \\
\hline & Governmental employee & 51 & $24.1 \%$ \\
\hline & Private/NGO employee & 43 & $20.3 \%$ \\
\hline & Farmer & 5 & $2.4 \%$ \\
\hline
\end{tabular}




\begin{tabular}{|llll|}
\hline Variables Category & Frequency & Percentage \\
\hline & Housewife/unemployed & 47 & $22.2 \%$ \\
\hline
\end{tabular}

\section{Most effective preventive measure of COVID-19}

Most participants (86.8\%) responded "Cover mouth nose with facemask" is the most effective preventive measure of COVID-19. Study participants' responses on preventive measures such as "stay at home" and "use disinfectant" were $77.4 \%, 76.4 \%$ respectively (Table 3 ).

Table 3

Respondents' awareness on COVID-19 preventive measure, Addis Ababa, Ethiopia. May 2021.

\begin{tabular}{|c|c|c|c|}
\hline Variables Category & & Frequency & Percentage \\
\hline \multirow[t]{2}{*}{ Stay at home } & No & 48 & $22.6 \%$ \\
\hline & Yes & 164 & $77.4 \%$ \\
\hline \multirow[t]{2}{*}{ Maintain physical distancing } & No & 87 & $41.0 \%$ \\
\hline & Yes & 125 & $59.0 \%$ \\
\hline \multirow[t]{2}{*}{ Avoid close contact } & No & 85 & $40.1 \%$ \\
\hline & Yes & 127 & $59.9 \%$ \\
\hline \multirow[t]{2}{*}{ Cover mouth nose with facemask } & No & 28 & $13.2 \%$ \\
\hline & Yes & 184 & $86.8 \%$ \\
\hline \multirow[t]{2}{*}{ Frequent handwashing with soap } & No & 43 & $20.3 \%$ \\
\hline & Yes & 169 & $79.7 \%$ \\
\hline \multirow[t]{2}{*}{ Avoid touching of eyes nose and mouth with unwashed hands } & No & 68 & $32.1 \%$ \\
\hline & Yes & 144 & $67.9 \%$ \\
\hline \multirow[t]{2}{*}{ Avoid mass gathering } & No & 92 & $43.4 \%$ \\
\hline & Yes & 120 & $56.6 \%$ \\
\hline \multirow[t]{2}{*}{ Restrict movement } & No & 98 & $46.2 \%$ \\
\hline & Yes & 114 & $53.8 \%$ \\
\hline \multirow[t]{2}{*}{ Use disinfectant } & No & 50 & $23.6 \%$ \\
\hline & Yes & 162 & $76.4 \%$ \\
\hline
\end{tabular}

\section{The financial burden of COVID-19}


The most costly COVID-19 preventive measures that cause financial burden to the patients were costs for buying facemasks [135 (63.7\%)], disinfectants [117(55.2\%)], sops for handwashing [47 (22.2\%)] (Table 4).

Table 4

Respondents' financial burden of COVID-19 preventive measures, Addis Ababa, Ethiopia May 2021.

\begin{tabular}{|lllll|}
\hline Variables Category & & Frequency & Percentage \\
\hline Facemask & No & 77 & $36.3 \%$ \\
\cline { 2 - 5 } & Yes & 135 & $63.7 \%$ \\
\cline { 2 - 5 } & Noap for frequent hand washing & 165 & $77.8 \%$ \\
\cline { 2 - 4 } & Nes & 47 & $22.2 \%$ \\
\hline & No & 95 & $44.8 \%$ \\
\hline & Yes & 117 & $55.2 \%$ \\
\hline
\end{tabular}

\section{HIV care and treatment services during COVID-19}

Participants who obliged to chage health centre were 3 (1.4\%), and 27 (12.7\%) denied health services. Almost all participants said health care providers were polite and respectful (99.5\%), willing to listen and answer their questions (99.5\%), give attention to their individual needs (99.1\%) (Table 5). 
Table 5

Response of study participants on health care facility and service delivery, Addis Ababa, Ethiopia May 2021.

\begin{tabular}{|c|c|c|c|}
\hline Variables Category & & Frequency & Percentage \\
\hline \multirow[t]{2}{*}{ Obliged to change the health centre because of this pandemic? } & Yes & 3 & $1.4 \%$ \\
\hline & No & 209 & $98.6 \%$ \\
\hline \multirow[t]{2}{*}{ Denied health services? } & Yes & 27 & $12.7 \%$ \\
\hline & No & 185 & $87.3 \%$ \\
\hline \multirow[t]{2}{*}{ Politeness \& respect of health professionals? } & Yes & 211 & $99.5 \%$ \\
\hline & No & 1 & $0.5 \%$ \\
\hline \multirow{2}{*}{$\begin{array}{l}\text { Willingness of professionals to listen and } \\
\text { answer your questions? }\end{array}$} & Yes & 211 & $99.5 \%$ \\
\hline & No & 1 & $0.5 \%$ \\
\hline \multirow[t]{2}{*}{ Attention of professionals to your individual needs? } & Yes & 210 & $99.1 \%$ \\
\hline & No & 2 & $0.9 \%$ \\
\hline \multirow[t]{2}{*}{ Staff seemed uncomfortable with you? } & Yes & 23 & $10.8 \%$ \\
\hline & No & 189 & $89.2 \%$ \\
\hline \multirow{2}{*}{$\begin{array}{l}\text { Contact care provider when there is a health problem or } \\
\text { comorbidities quickly? }\end{array}$} & Yes & 101 & $47.6 \%$ \\
\hline & No & 111 & $52.4 \%$ \\
\hline
\end{tabular}

\section{Main barriers to access health care during the pandemic}

Among study subjects, 189 (89.2\%) said transport disruption was the main barrier to access health care. Fear of getting infected with COVID-19 (78.8\%) was the second main barrier for the participants [figure 1].

\section{COVID-19 precaution measures in healthcare facilities}

Among participants, 143 (67.5\%) responded that health centers provide screening services for COVID-19 and all health professionals wear masks. Participants responded that there was water (97.2\%) and soap (95.8\%) at the gate of the healthcare facilities, but not sanitizer (74.1\%) (Table 6). 
Table 6

Response of study participants on health facilities precautions for COVID-19 protection, Addis Ababa, Ethiopia, May 2021.

\begin{tabular}{|c|c|c|c|}
\hline Variables Category & & Frequency & Percentage \\
\hline \multirow[t]{2}{*}{ Health centre provide screening service for COVID-19? } & Yes & 143 & $67.5 \%$ \\
\hline & No & 69 & $32.5 \%$ \\
\hline \multirow[t]{2}{*}{ Health professionals wear the gloves during caregiving? } & Yes & 211 & $99.5 \%$ \\
\hline & No & 1 & $0.5 \%$ \\
\hline \multirow{2}{*}{$\begin{array}{l}\text { Health professionals wear the mask during } \\
\text { caregiving? }\end{array}$} & Yes & 212 & $100 \%$ \\
\hline & No & 0 & $0.0 \%$ \\
\hline \multirow{2}{*}{$\begin{array}{l}\text { Water available at the entrance of the health centre for hand } \\
\text { washing? }\end{array}$} & Yes & 206 & $97.2 \%$ \\
\hline & No & 6 & $2.8 \%$ \\
\hline \multirow{2}{*}{$\begin{array}{l}\text { Soap available at the entrance of the health centre for hand } \\
\text { washing? }\end{array}$} & Yes & 203 & $95.8 \%$ \\
\hline & No & 9 & $4.2 \%$ \\
\hline \multirow{2}{*}{$\begin{array}{l}\text { Sanitizer available at the entrance of the } \\
\text { health centre for cleaning of hands? }\end{array}$} & Yes & 55 & $25.9 \%$ \\
\hline & No & 157 & $74.1 \%$ \\
\hline
\end{tabular}

\section{Medications and follow-ups during COVID-19}

Among the total participants, 125 (59.0\%) said ordered drugs are available. Two hundred (94.3\%) were able to collect their multi-month drug supply. Participants who missed appointments, follow-up tests, and counseling services were 58 (27.4\%), 56 (26.4\%), and 55 (25.9\%) respectively (Table 7). 
Table 7

Response of study participants on medications and follow-up, Addis Ababa, Ethiopia May, 2021.

\begin{tabular}{|c|c|c|c|}
\hline Variables Category & & Frequency & Percentage \\
\hline \multirow[t]{3}{*}{ Availability of ordered drugs? } & Yes & 125 & $59.0 \%$ \\
\hline & Some & 80 & $37.7 \%$ \\
\hline & Not at all & 7 & $3.3 \%$ \\
\hline \multirow[t]{4}{*}{ Non-medical support since COVID19? } & Same as before & 163 & $76.9 \%$ \\
\hline & Slightly harder & 15 & $7.1 \%$ \\
\hline & Much harder & 23 & $10.8 \%$ \\
\hline & Impossible & 11 & $5.2 \%$ \\
\hline \multirow[t]{2}{*}{ Have you had multi-month drug supply } & Yes & 200 & $94.3 \%$ \\
\hline & No & 12 & $5.7 \%$ \\
\hline \multirow[t]{2}{*}{ For how many months } & 3 months & 90 & $42.5 \%$ \\
\hline & 6 monthes & 110 & $51.9 \%$ \\
\hline \multirow[t]{2}{*}{ Have you missed appointments (visits) } & Yes & 58 & $27.4 \%$ \\
\hline & No & 154 & $72.6 \%$ \\
\hline \multirow[t]{2}{*}{ Follow-up tests done } & Yes & 156 & $73.6 \%$ \\
\hline & No & 56 & $26.4 \%$ \\
\hline \multirow{2}{*}{$\begin{array}{l}\text { Counselling done on your medication or health } \\
\text { status? }\end{array}$} & Yes & 157 & $74.1 \%$ \\
\hline & No & 55 & $25.9 \%$ \\
\hline
\end{tabular}

\section{Logistic Regression analysis of missing appointments/visits for medication refill variable}

By Bivariate and Multivariate Logistic Regression analysis of missing appointments/visits for medication refill variable, independent variables such as age, education, fear of COVID-19, transport disruption, reduced income, unable to access mask, sanitizer available, multi months drug supply, cost of disinfectant, non medical support since COVID-19 were significantly associated (Table 8). 
Table 8

Bivariate and Multivariate Logistic Regression analysis of missing appointments/visits for medication refill variable, Addis Ababa, Ethiopia, 2021.

\begin{tabular}{|c|c|c|c|c|c|c|}
\hline \multirow[b]{2}{*}{ Variables Category } & & \multicolumn{2}{|c|}{$\begin{array}{l}\text { Missed } \\
\text { appointments }\end{array}$} & \multicolumn{2}{|l|}{ Odds ratio } & \multirow[b]{2}{*}{$\begin{array}{l}\mathrm{P} \\
\text { value }\end{array}$} \\
\hline & & No & Yes & $\operatorname{COR}(\mathrm{Cl})$ & $\mathrm{AOR}(\mathrm{Cl})$ & \\
\hline \multirow[t]{3}{*}{ Age } & $18-34$ & $\begin{array}{l}43 \\
(28.0 \%)\end{array}$ & $\begin{array}{l}12 \\
(20.7 \%)\end{array}$ & 1 & $011<001$ & 0.252 \\
\hline & $35-54$ & $\begin{array}{l}92 \\
(59.7 \%)\end{array}$ & $\begin{array}{l}11 \\
(19.0 \%)\end{array}$ & $\begin{array}{l}0.43(0.175- \\
1.048)\end{array}$ & $\begin{array}{l}0.41(0.091- \\
1.875)\end{array}$ & \multirow{2}{*}{$0.013^{*}$} \\
\hline & $\geq 55$ & $\begin{array}{l}19 \\
(12.3 \%)\end{array}$ & $\begin{array}{l}35 \\
(60.3 \%)\end{array}$ & $\begin{array}{l}6.60(2.823- \\
15.434)\end{array}$ & $\begin{array}{l}6.73(1.495- \\
30.310)\end{array}$ & \\
\hline \multirow[t]{7}{*}{ Education } & $\begin{array}{l}\text { No } \\
\text { education }\end{array}$ & $\begin{array}{l}14 \\
(9.1 \%)\end{array}$ & $\begin{array}{l}31 \\
(53.5 \%)\end{array}$ & 1 & 1 & \multirow{2}{*}{$\begin{array}{l}0.001 * \\
0.002^{*}\end{array}$} \\
\hline & \multirow{2}{*}{$\begin{array}{l}\text { Read + } \\
\text { write }\end{array}$} & \multirow{2}{*}{$\begin{array}{l}19 \\
(12.3 \%)\end{array}$} & \multirow{2}{*}{$\begin{array}{l}11 \\
(19.0 \%)\end{array}$} & $\begin{array}{l}0.25(0.096- \\
0.670)\end{array}$ & $\begin{array}{l}0.01(0.001- \\
0.165)\end{array}$ & \\
\hline & & & & \multirow{2}{*}{$\begin{array}{l}0.08(0.031- \\
0.203)\end{array}$} & \multirow{2}{*}{$\begin{array}{l}0.02(0.002- \\
0.229)\end{array}$} & 0.052 \\
\hline & $\begin{array}{l}\text { Primary } \\
\text { edu. }\end{array}$ & $\begin{array}{l}50 \\
(32.5 \%)\end{array}$ & $\begin{array}{l}9 \\
(15.5 \%)\end{array}$ & & & \multirow[t]{4}{*}{0.997} \\
\hline & \multirow{2}{*}{$\begin{array}{l}\text { Secondary } \\
\text { edu. }\end{array}$} & \multirow{2}{*}{$\begin{array}{l}38 \\
(24.7 \%)\end{array}$} & \multirow{2}{*}{$\begin{array}{l}6 \\
(10.3 \%)\end{array}$} & $\begin{array}{l}0.07(0.024- \\
0.201)\end{array}$ & $\begin{array}{l}0.05(0.003- \\
1.022)\end{array}$ & \\
\hline & & & & \multirow{2}{*}{$\begin{array}{l}0.01 \\
(0.0012- \\
1.021)\end{array}$} & \multirow{2}{*}{$\begin{array}{l}0.01(0.001- \\
1.002)\end{array}$} & \\
\hline & $\geq$ Diploma & $(21.4 \%)$ & $(1.7 \%)$ & & & \\
\hline \multirow[t]{2}{*}{ Fear of COVID- 19} & No & $\begin{array}{l}44 \\
(28.6 \%)\end{array}$ & $\begin{array}{l}1 \\
(1.7 \%)\end{array}$ & \multirow{2}{*}{$\begin{array}{l}1 \\
22.80 \\
(3.062- \\
169.782)\end{array}$} & 1 & $0.004^{*}$ \\
\hline & Yes & $\begin{array}{l}110 \\
(71.4 \%)\end{array}$ & $\begin{array}{l}57 \\
(98.3 \%)\end{array}$ & & $\begin{array}{l}24.93 \\
(2.798- \\
222.279)\end{array}$ & \\
\hline Transport disruption & No & $\begin{array}{l}22 \\
(14.3 \%)\end{array}$ & $\begin{array}{l}1 \\
(1.7 \%)\end{array}$ & 1 & nnounat & $0.038^{*}$ \\
\hline & Yes & $\begin{array}{l}132 \\
(85.7 \%)\end{array}$ & $\begin{array}{l}57 \\
(98.3 \%)\end{array}$ & $\begin{array}{l}9.50(1.250- \\
31.185)\end{array}$ & $\begin{array}{l}4.90(1.031- \\
23.174)\end{array}$ & \\
\hline Reduced income & No & $\begin{array}{l}53 \\
(34.4 \%)\end{array}$ & $\begin{array}{l}3 \\
(5.2 \%)\end{array}$ & 1 & 1 & $0.026^{*}$ \\
\hline & Yes & $\begin{array}{l}101 \\
(65.6 \%)\end{array}$ & $\begin{array}{l}55 \\
(94.8 \%)\end{array}$ & $\begin{array}{l}9.62(2.873- \\
32.219)\end{array}$ & $\begin{array}{l}5.64(1.234- \\
25.812)\end{array}$ & \\
\hline Unable to access & No & 64 & 2 & 1 & 1 & $0.024^{*}$ \\
\hline & & & & $\begin{array}{l}19.91 \\
(4.687-\end{array}$ & $\begin{array}{l}7.67(1.303- \\
45.174)\end{array}$ & \\
\hline
\end{tabular}




\begin{tabular}{|c|c|c|c|c|c|c|}
\hline & & \multicolumn{2}{|c|}{$\begin{array}{l}\text { Missed } \\
\text { appointments }\end{array}$} & \multicolumn{3}{|l|}{ Odds ratio } \\
\hline & Yes & $\begin{array}{l}90 \\
(58.4 \%)\end{array}$ & $\begin{array}{l}56 \\
(96.6 \%)\end{array}$ & & & \\
\hline \multirow[t]{4}{*}{ Sanitizer available } & No & 110 & 47 & 1 & 1 & $0.026 *$ \\
\hline & Yes & & & & & \\
\hline & & $(71.4 \%)$ & $(81.0 \%)$ & \multirow{2}{*}{$\begin{array}{l}0.58(0.278- \\
1.231)\end{array}$} & \multirow{2}{*}{$\begin{array}{l}0.07(0.007- \\
0.729)\end{array}$} & \\
\hline & & $\begin{array}{l}44 \\
(28.6 \%)\end{array}$ & $\begin{array}{l}11 \\
(19.0 \%)\end{array}$ & & & \\
\hline \multirow[t]{2}{*}{ For how many months } & 3 months & $\begin{array}{l}52 \\
(35.9 \%)\end{array}$ & $\begin{array}{l}38 \\
(69.1 \%)\end{array}$ & \multirow{2}{*}{$\begin{array}{l}1 \\
0.25(0.129- \\
0.486)\end{array}$} & \multirow{2}{*}{$\begin{array}{l}1 \\
0.33(0.132- \\
0.825)\end{array}$} & \multirow[t]{2}{*}{$0.018^{*}$} \\
\hline & 6 months & $\begin{array}{l}93 \\
(64.1 \%)\end{array}$ & $\begin{array}{l}17 \\
(30.9 \%)\end{array}$ & & & \\
\hline \multirow[t]{2}{*}{ Cost of disinfectant } & No & 85 & 10 & 1 & 1 & $0.023 *$ \\
\hline & Yes & $\begin{array}{l}69 \\
(44.8 \%)\end{array}$ & $\begin{array}{l}48 \\
(82.8 \%)\end{array}$ & $\begin{array}{l}5.91(2.788- \\
12.539)\end{array}$ & $\begin{array}{l}16.64 \\
(1.462- \\
189.569)\end{array}$ & \\
\hline \multirow[t]{6}{*}{$\begin{array}{l}\text { Non-medical support } \\
\text { since COVID-19 }\end{array}$} & $\begin{array}{l}\text { Same as } \\
\text { before }\end{array}$ & $\begin{array}{l}130 \\
(84.4 \%)\end{array}$ & $\begin{array}{l}33 \\
(56.9 \%)\end{array}$ & 1 & 1 & 0.233 \\
\hline & \multirow{2}{*}{$\begin{array}{l}\text { Slightly } \\
\text { harder }\end{array}$} & \multirow{2}{*}{$\begin{array}{l}12 \\
(7.8 \%)\end{array}$} & \multirow{2}{*}{$\begin{array}{l}3 \\
(5.2 \%)\end{array}$} & $\begin{array}{l}0.98(0.263- \\
3.693)\end{array}$ & $\begin{array}{l}3.68(0.434- \\
31.204)\end{array}$ & 0.100 \\
\hline & & & & \multirow{2}{*}{$\begin{array}{l}5.12(2.064- \\
12.705)\end{array}$} & \multirow{2}{*}{$\begin{array}{l}3.780 .774- \\
18.421)\end{array}$} & \multirow{4}{*}{$0.044^{*}$} \\
\hline & \multirow{2}{*}{$\begin{array}{l}\text { Much } \\
\text { harder }\end{array}$} & \multirow{3}{*}{$\begin{array}{l}10 \\
(6.5 \%) \\
2 \\
(1.3 \%)\end{array}$} & \multirow{2}{*}{$\begin{array}{l}13 \\
(22.4 \%)\end{array}$} & & & \\
\hline & & & & 17.72 & $2.32(1.547-$ & \\
\hline & Impossible & & $(15.5 \%)$ & $85.987)$ & & \\
\hline
\end{tabular}

*Statistically significant at $p$-value $<0.05, \mathrm{COR}=$ crude odds ratio at $95 \%$ confidence interval; $A O R=$ adjusted odds ratio at $95 \%$ confidence interval.

\section{Logistic Regression analysis of follow-up tests variable}

In Bivariate and Multivariate Logistic Regression analysis of follow-up tests variable, the following variables found to be significant: age, denied health services, reduced income/ money to travel, partial lockdown and unable to access face mask(Table 9). 
Table 9

Bivariate and Multivariate Logistic Regression analysis of follow-up tests variable, Addis Ababa, Ethiopia, 2021.

\begin{tabular}{|c|c|c|c|c|c|c|}
\hline \multirow[b]{2}{*}{ Variables Category } & \multicolumn{2}{|c|}{ Follow up test } & \multicolumn{2}{|c|}{ Odds ratio } & & \\
\hline & No & Yes & \multicolumn{2}{|c|}{$\operatorname{coR}(\mathrm{Cl})$} & \multicolumn{2}{|c|}{$P$ value } \\
\hline \multirow[t]{5}{*}{ Age } & \multirow{2}{*}{$\begin{array}{l}18- \\
34\end{array}$} & \multirow{2}{*}{$\begin{array}{l}12 \\
(21.4 \%)\end{array}$} & \multirow{2}{*}{$\begin{array}{l}43 \\
(27.6 \%)\end{array}$} & 1 & 1 & 0.073 \\
\hline & & & & $2.59(1.041-$ & \multirow{2}{*}{$\begin{array}{l}2.65(0.913- \\
7.670)\end{array}$} & \multirow[t]{4}{*}{$0.004^{*}$} \\
\hline & \multirow{2}{*}{$\begin{array}{l}35- \\
54\end{array}$} & \multirow{2}{*}{$\begin{array}{l}10 \\
(17.9 \%)\end{array}$} & \multirow{2}{*}{$\begin{array}{l}93 \\
(59.6 \%)\end{array}$} & 6.472) & & \\
\hline & & & & $0.16(0.070-$ & \multirow{2}{*}{$\begin{array}{l}0.22(0.076- \\
0.621)\end{array}$} & \\
\hline & $\geq 55$ & $\begin{array}{l}34 \\
(60.7 \%)\end{array}$ & $\begin{array}{l}20 \\
(12.8 \%)\end{array}$ & & & \\
\hline \multirow[t]{3}{*}{ Partial lockdown } & No & \multirow{3}{*}{$\begin{array}{l}1(1.8 \%) \\
55 \\
(98.2 \%)\end{array}$} & \multirow{2}{*}{$\begin{array}{l}49 \\
(31.4 \%)\end{array}$} & 1 & 1 & \multirow[t]{3}{*}{$0.034 *$} \\
\hline & & & & $0.04(0.005-$ & $0.10(0.011-$ & \\
\hline & Yes & & $\begin{array}{l}107 \\
(68.6 \%)\end{array}$ & $0.295)$ & $0.833)$ & \\
\hline \multirow{3}{*}{$\begin{array}{l}\text { Denied health } \\
\text { services }\end{array}$} & No & \multirow{2}{*}{$\begin{array}{l}35 \\
(62.5 \%)\end{array}$} & \multirow{2}{*}{$\begin{array}{l}150 \\
(96.2 \%)\end{array}$} & 1 & 1 & \multirow[t]{3}{*}{$0.001 *$} \\
\hline & & & & $0.07(0.025-$ & $0.15(0.045-$ & \\
\hline & Yes & $\begin{array}{l}21 \\
(37.5 \%)\end{array}$ & $6(3.8 \%)$ & $0.177)$ & $0.475)$ & \\
\hline \multirow[t]{3}{*}{ Reduced income } & No & \multirow{3}{*}{$\begin{array}{l}3(5.4 \%) \\
53 \\
(94.6 \%)\end{array}$} & \multirow{2}{*}{$\begin{array}{l}53 \\
(34.0 \%)\end{array}$} & 1 & 1 & \multirow[t]{3}{*}{$0.023 *$} \\
\hline & & & & $0.11(0.033-$ & $0.18(0.039-$ & \\
\hline & Yes & & $\begin{array}{l}103 \\
(66.0 \%)\end{array}$ & $0.369)$ & $0.784)$ & \\
\hline \multirow{2}{*}{$\begin{array}{l}\text { Unable to get } \\
\text { mask }\end{array}$} & No & $2(3.6 \%)$ & $\begin{array}{l}64 \\
(41.0 \%)\end{array}$ & 1 & 1 & \multirow[t]{2}{*}{$0.006^{*}$} \\
\hline & Yes & $\begin{array}{l}54 \\
(96.4 \%)\end{array}$ & $\begin{array}{l}92 \\
(59.0 \%)\end{array}$ & $\begin{array}{l}0.05(0.013- \\
0.226)\end{array}$ & $\begin{array}{l}0.12(0.026- \\
0.543)\end{array}$ & \\
\hline
\end{tabular}

*Statistically significant at $\mathrm{p}$-value $<0.05, \mathrm{COR}=$ crude odds ratio at $95 \%$ confidence interval; $A O R=$ adjusted odds ratio at $95 \%$ confidence interval.

\section{Logistic Regression analysis of counseling variable}

Bivariate and Multivariate Logistic Regression analysis of counseling variable, factors such as age, education, fear of COVID-19, reduced income money to travel, unable to access face mask and partial lockdown were significant(Table 10). 
Table 10

Bivariate and Multivariate Logistic Regression analysis of counseling variable, Addis Ababa, Ethiopia, 2021.

\begin{tabular}{|c|c|c|c|c|c|c|}
\hline \multirow{2}{*}{ Variable Category } & & \multicolumn{2}{|c|}{ Counsling done } & \multicolumn{3}{|l|}{ Odds ratio } \\
\hline & & No & Yes & $\operatorname{COR}(\mathrm{Cl})$ & $\mathrm{AOR}(\mathrm{Cl})$ & $\begin{array}{l}P \\
\text { value }\end{array}$ \\
\hline \multirow[t]{3}{*}{ Age } & $18-34$ & $\begin{array}{l}12 \\
(21.8 \%)\end{array}$ & $\begin{array}{l}43 \\
(27.4 \%)\end{array}$ & 1 & 1 & 0.105 \\
\hline & $35-54$ & 10 & 93 & $\begin{array}{l}2.59(1.041- \\
6.472)\end{array}$ & $\begin{array}{l}2.28(0.842- \\
6.170)\end{array}$ & $0.002^{*}$ \\
\hline & $\geq 55$ & $\begin{array}{l}33 \\
(60.0 \%)\end{array}$ & $\begin{array}{l}21 \\
(13.4 \%)\end{array}$ & $\begin{array}{l}0.18(0.077- \\
0.412)\end{array}$ & $\begin{array}{l}0.21(0.078- \\
0.570)\end{array}$ & \\
\hline \multirow[t]{7}{*}{ Education } & $\begin{array}{l}\text { No } \\
\text { education }\end{array}$ & $\begin{array}{l}29 \\
(52.7 \%)\end{array}$ & $\begin{array}{l}16 \\
(10.2 \%)\end{array}$ & 1 & 1 & \multirow{2}{*}{$\begin{array}{l}0.020 * \\
0.000 *\end{array}$} \\
\hline & \multirow{2}{*}{$\begin{array}{l}\text { Read + } \\
\text { write }\end{array}$} & \multirow{2}{*}{$\begin{array}{l}11 \\
(20.0 \%)\end{array}$} & \multirow{2}{*}{$\begin{array}{l}19 \\
(12.1 \%)\end{array}$} & $\begin{array}{l}3.24(1.241- \\
8.449)\end{array}$ & $\begin{array}{l}3.68(1.230- \\
11.022)\end{array}$ & \\
\hline & & & & \multirow{2}{*}{$\begin{array}{l}11.95(4.572- \\
31.251)\end{array}$} & \multirow{2}{*}{$\begin{array}{l}11.46(3.906- \\
33.615)\end{array}$} & \multirow{2}{*}{$\begin{array}{l}0.003^{*} \\
0.801\end{array}$} \\
\hline & \multirow{2}{*}{$\begin{array}{l}\text { Primary } \\
\text { edu. }\end{array}$} & $\begin{array}{l}8 \\
(14.5 \%)\end{array}$ & $\begin{array}{l}51 \\
(32.5 \%)\end{array}$ & & & \\
\hline & & \multirow{2}{*}{$\begin{array}{l}6 \\
(10.9 \%)\end{array}$} & \multirow{2}{*}{$\begin{array}{l}38 \\
(24.2 \%)\end{array}$} & \multirow{2}{*}{$\begin{array}{l}11.87(4.142- \\
34.047)\end{array}$} & $\begin{array}{l}6.48(1.921- \\
21.876)\end{array}$ & \\
\hline & $\begin{array}{l}\text { Secondary } \\
\text { edu. }\end{array}$ & & & & \multirow{2}{*}{$\begin{array}{l}1.23(0.238- \\
6.412)\end{array}$} & \\
\hline & $\geq$ Diploma & $\begin{array}{l}1 \\
(1.8 \%)\end{array}$ & $\begin{array}{l}33 \\
(21.0 \%)\end{array}$ & $\begin{array}{l}4.60(0.391- \\
15.227)\end{array}$ & & \\
\hline \multirow{2}{*}{$\begin{array}{l}\text { Fear of COVID- } \\
19\end{array}$} & No & $\begin{array}{l}1 \\
(1.8 \%)\end{array}$ & $\begin{array}{l}44 \\
(28.0 \%)\end{array}$ & 1 & 1 & \multirow[t]{2}{*}{$0.041 *$} \\
\hline & Yes & $\begin{array}{l}54 \\
(98.2 \%)\end{array}$ & $\begin{array}{l}113 \\
(72.0 \%)\end{array}$ & $\begin{array}{l}0.05(0.006- \\
0.354)\end{array}$ & $\begin{array}{l}0.11(0.013- \\
0.912)\end{array}$ & \\
\hline \multirow[t]{2}{*}{$\begin{array}{l}\text { Reduced } \\
\text { income }\end{array}$} & No & $\begin{array}{l}3 \\
(5.5 \%)\end{array}$ & $\begin{array}{l}53 \\
(33.8 \%)\end{array}$ & \multirow{2}{*}{$\begin{array}{l}1 \\
0.11(0.034- \\
0.380)\end{array}$} & 1 & \multirow[t]{2}{*}{$0.014^{*}$} \\
\hline & Yes & $\begin{array}{l}52 \\
(94.5 \%)\end{array}$ & $\begin{array}{l}104 \\
(66.2 \%)\end{array}$ & & $\begin{array}{l}0.17(0.041- \\
0.699)\end{array}$ & \\
\hline \multirow{2}{*}{$\begin{array}{l}\text { Unable get } \\
\text { face mask }\end{array}$} & No & $\begin{array}{l}2 \\
(3.6 \%)\end{array}$ & $\begin{array}{l}64 \\
(40.8 \%)\end{array}$ & 1 & 1 & $0.044^{*}$ \\
\hline & Yes & $\begin{array}{l}53 \\
(96.4 \%)\end{array}$ & $\begin{array}{l}93 \\
(59.2 \%)\end{array}$ & $\begin{array}{l}0.05(0.013- \\
0.233)\end{array}$ & $\begin{array}{l}0.19(0.039- \\
0.959)\end{array}$ & \\
\hline Partial & No & 1 & 49 & 1 & 1 & $0.031^{*}$ \\
\hline & & & & $\begin{array}{l}0.04(0.005- \\
0.304)\end{array}$ & $\begin{array}{l}0.08(0.008- \\
0.790)\end{array}$ & \\
\hline
\end{tabular}




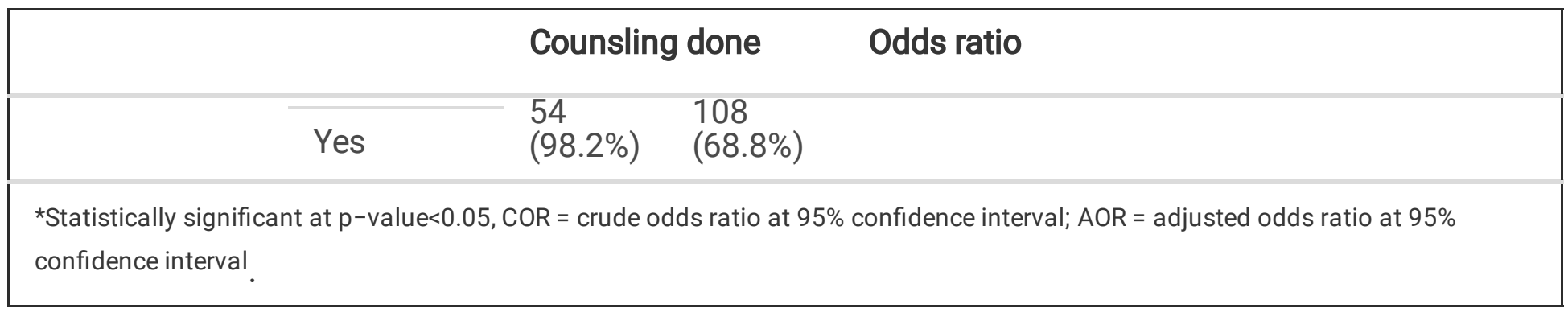

\section{Discussions}

To the best of our knowledge, this study was the first of its kind to assess the impact of COVID-19 on HIV care and treatment services in Ethiopia. We studied the overlap between two ongoing pandemics (HIV and COVID-19) in Ethiopia. The findings underscore several factors rendering HIV care and treatment services more difficult. A significant number of participants have missed appointments, follow-up tests, and counseling services due to COVID-19. COVID-19 containment measures taken by the government, patients' sociodemographic characteristics, inconsistent access to personal protective equipment were the main factors that have hindered HIV patients' retention and adherence to their routine HIV care and treatment.

The patient living with HIV had great concerns about whether they are at high risk for the pandemic and the worse outcomes if they get infected with COVID-19. Research findings on these concerns have been in agreement with previous studies conducted elsewhere [18, 19, 33-36]. Studies indicated that though the pandemic affected the health care for all disease conditions, chronic patients such as people living with HIV are likely to be uniquely vulnerable $[4,37,38]$. In our findings, those who had formal education are more likely to have care and treatment services. This might be because respondents who had formal education may have a deeper understanding of the negative consequence if they missed their follow-up visits and they could have more tendency to request and access information about COVID-19 and its preventive measures.

Our results also indicated that HIV patients who had a fear of getting infected with COVID-19 and those who were elderly were more likely to miss appointments for care and treatment. This is also consistent with other findings [39-41]. It has been reported that the elderly and people with chronic conditions are more likely to be infected with COVID-19 and HIV patients may miss appointments as a result. COVID-19 containment measures taken in Ethiopia had a significant contribution in halting the spread of COVID-19 in Ethiopia; however, they had their own implications on HIV care and treatment services as the response from the HIV patients indicated. Transport disruption, partial lockdown that impaired mobility, and income reduction were significant factors for missing health care visits, which was in agreement with previous studies conducted in Ethiopia [10, 42, ] and elsewhere in the world [43-50] that COVID-19 containment measure had a significant impact on patients' access to healthcare facilities.

Undue expenses related to protective equipment including face masks sanitizers were a burden for the people living with HIV. The city of Addis Ababa introduced innovative measures providing ART 
medications for 3-6 months to mitigate these challenges. In our finding those who collect medications for 6 months were less likely to miss appointments for medication refill compared to those who took for 3 months.

Indirect impacts arising from the pandemic which reduced non-medical support had economical burdens. Indeed, health centers in Addis Ababa have had preeminent COVID-19 precaution procedures and measures to protect their clients from the pandemic. Availability of sanitizer, water, and soap at the health facilities' gates encouraged the HIV patients to attend their routine care.

Our study has some limitations. The study was limited to healthcare facilities in Addis Ababa, and therefore may not be representative of Ethiopia. As the study design was a cross-sectional study, it does not show a causal relationship and only provides a view of the impacts of COVID-19 in a specific period. Otherwise, the study was based on real-time, patient-level primary data and it was conducted in a resource-constrained, high HIV burden country context.

\section{Conclusion}

COVID-19 had a significant burden on HIV patients to attend their routine clinical care and treatment, which may lead to treatment failure and drug resistance. The impact was on their appointments for medication refills and clinical and laboratory follow-ups. Targeted initiatives are needed to sustain HIV clinical care and treatment services and improve the wellbeing of people living with HIV. Stakeholders such as the Addis Ababa health bureau, the ministry of health, and others should work in partnership to reduce the impact of this pandemic on those patients maintain their economic well-being.

\section{Abbreviations}

AAU, Addis Ababa University; COVID-19, Coronavirus Disease 2019; SARS-CoV-2, Severe acute respiratory syndrome coronavirus 2; HIV, Human Immunodeficiency Virus; PLWH, people living with HIV; HC, Health center; ICU, Intensive care unit; PPE, Personal protective equipment; WHO, World health organization; AOR, Adjusted Odds Ratio; Cl, Confidence Interval.

\section{Declarations}

\section{Acknowledgments}

The authors acknowledge the Center for Innovative Drug Development and Therapeutic Trials for Africa (CDT-Africa), College of Health Sciences, Addis Ababa University, for supporting the study. The authors acknowledge all health centers from where data were collected and study participants for their cooperation. The authors also forward their gratitude to Amarech Taye for her cooperation during the data collection.

\section{Ethics declarations}


Ethical clearance was obtained from the Scientific and Ethics Review Committee of the Center for Innovative Drug Development and Therapeutic Trials for Africa (CDT-Africa), College of Health Sciences, Addis Ababa University. Ethical clearance and support letters were obtained from Addis Ababa public health research and emergency directorate, Addis Ababa City Government Health Bureau. Informed consent was obtained from the study participants and their privacy and confidentiality were maintained strictly.

\section{Consent for publication}

Not applicable.

\section{Competing of interest}

The authors declare that they have no competing interests.

\section{Contributions}

DC collected the primary data, conducted the analyses, and draft the manuscript. TM and YW contributed to the data collection and analysis and reviewed the manuscript. All authors have read and approved the manuscript.

\section{Funding}

This study was supported by the Center for Innovative Drug Development and Therapeutic Trials for Africa (CDT-Africa). TM was supported in part by the Fogarty International Center and National Institute of Allergy and Infectious Diseases of the US National Institutes of Health (D43TW009127). The content is solely the responsibility of the authors and does not necessarily represent the official views of the CDTAfrica or the National Institutes of Health.

\section{References}

1. World Health Organization. Coronavirus disease 2019 (COVID-19) dashboard. Geneva; 2021.

2. Mirzaei H, McFarland W, Karamouzian M, Sharifi H. COVID-19 Among People Living with HIV: A Systematic Review. AIDS Behav. 2021;25(1):85-92.

3. Lesko CR, Bengtson AM. HIV and COVID-19: Intersecting Epidemics With Many Unknowns. Am J Epidemiol. 2021;190(1):10-6.

4. Jones DL, Morgan KE, Martinez PC, Rodriguez VJ, Vazquez A, Raccamarich PD, Alcaide ML. COVID19 Burden and Risk Among People With HIV. J Acquir Immune Defic Syndr. 2021 Jun 1;87(2):869874.

5. Zang X, Krebs E, Chen S, et al. The Potential Epidemiological Impact of Coronavirus Disease 2019 (COVID-19) on the Human Immunodeficiency Virus (HIV) Epidemic and the Cost-effectiveness of Linked, Opt-out HIV Testing: A Modeling Study in 6 US Cities. Clin Infect Dis. 2021;72(11):e828-34. 
6. Gatechompol S, Avihingsanon A, Putcharoen O, Ruxrungtham K, Kuritzkes DR. COVID-19 and HIV infection co-pandemics and their impact: a review of the literature. AIDS Res Ther. 2021;18(1):28. Published 2021 May 5.

7. Parmley LE, Hartsough K, Eleeza O, et al. COVID-19 preparedness at health facilities and community service points serving people living with HIV in Sierra Leone. PLoS One. 2021;16(4):e0250236. Published 2021 Apr 15.

8. Budak JZ, Scott JD, Dhanireddy S, Wood BR. The Impact of COVID-19 on HIV Care Provided via Telemedicine-Past, Present, and Future. Curr HIV/AIDS Rep. 2021;18(2):98-104.

9. Tamuzi JL, Ayele BT, Shumba CS, et al. Implications of COVID-19 in high burden countries for HIV/TB: A systematic review of evidence. BMC Infect Dis. 2020;20(1):744. Published 2020 Oct 9.

10. Mohammed H, Oljira L, Roba KT, Yimer G, Fekadu A, Manyazewal T. Containment of COVID-19 in Ethiopia and implications for tuberculosis care and research. Infect Dis Poverty. 2020;9(1):131. Published 2020 Sep 16.

11. Kassie BA, Adane A, Tilahun YT, Kassahun EA, Ayele AS, Belew AK. Knowledge and attitude towards COVID-19 and associated factors among health care providers in Northwest Ethiopia. PLoS One. 2020;15(8):e0238415. Published 2020 Aug 28.

12. Tesfaye ZT, Yismaw MB, Negash Z, Ayele AG. COVID-19-Related Knowledge, Attitude and Practice Among Hospital and Community Pharmacists in Addis Ababa, Ethiopia. Integr Pharm Res Pract. 2020;9:105-12. Published 2020 Aug 24.

13. Zikargae MH. COVID-19 in Ethiopia: Assessment of How the Ethiopian Government has Executed Administrative Actions and Managed Risk Communications and Community Engagement. Risk Manag Healthc Policy. 2020;13:2803-10. Published 2020 Dec 3.

14. Asnakew Z, Asrese K, Andualem M. Community Risk Perception and Compliance with Preventive Measures for COVID-19 Pandemic in Ethiopia. Risk Manag Healthc Policy. 2020;13:2887-97. Published 2020 Dec 8.

15. HIV.gov. Global statistics. 2019. Available at https://www.hiv.gov/hiv-basics/overview/data-andtrends/global-statistics (accessed 5 july 2021).

16. World Health Organization. summary of the global HIV epidemic. Geneva; 2019. Available from: https://www.who.int/gho/hiv/en/.

17. Huang J, Xie N, Hu X, et al. Epidemiological, virological and serological features of COVID-19 cases in people living with HIV in Wuhan City: A population-based cohort study [published online ahead of print, 2020 Aug 17]. Clin Infect Dis. 2020;ciaa1186.

18. Nagarakanti SR, Okoh AK, Grinberg S, Bishburg E. Clinical outcomes of patients with COVID-19 and HIV coinfection. J Med Virol. 2021;93(3):1687-93.

19. Mondi A, Cimini E, Colavita F, et al. COVID-19 in people living with HIV: Clinical implications of dynamics of the immune response to SARS-CoV-2. J Med Virol. 2021;93(3):1796-804.

20. Sachdev D, Mara E, Hsu L, et al. COVID-19 Susceptibility and Outcomes Among People Living With HIV in San Francisco. J Acquir Immune Defic Syndr. 2021;86(1):19-21. 
21. Seddiki N, French M. COVID-19 and HIV-Associated Immune Reconstitution Inflammatory Syndrome: Emergence of Pathogen-Specific Immune Responses Adding Fuel to the Fire. Front Immunol. 2021;12:649567. Published 2021 Mar 24.

22. hekkur P, Tweya H, Phiri S, et al. Assessing the Impact of COVID-19 on TB and HIV Programme Services in Selected Health Facilities in Lilongwe, Malawi: Operational Research in Real Time. Trop Med Infect Dis. 2021;6(2):81. Published 2021 May 19.

23. Liang M, Luo N, Chen M, et al. Prevalence and Mortality due to COVID-19 in HIV Co-Infected Population: A Systematic Review and Meta-Analysis [published online ahead of print, 2021 May 3]. Infect Dis Ther. 2021;1-19.

24. Riley ED, Hickey MD, Imbert E, Clemenzi-Allen AA, Gandhi M. Coronavirus Disease 2019 (COVID-19) and HIV Spotlight the United States Imperative for Permanent Affordable Housing. Clin Infect Dis. 2021;72(11):2042-3.

25. Bartilotti Matos F, Davies P. Pearls and pitfalls: Two contrasting HIV diagnoses in the COVID-19 era and the case for screening. J Med Virol. 2021;93(2):652-4.

26. Santos GM, Ackerman B, Rao A, et al. Economic, Mental Health, HIV Prevention and HIV Treatment Impacts of COVID-19 and the COVID-19 Response on a Global Sample of Cisgender Gay Men and Other Men Who Have Sex with Men. AIDS Behav. 2021;25(2):311-21.

27. Bhatt $M$, Soneja $M$, Gupta N. COVID-19 in patients living with human immunodeficiency virus (HIV) infection: Challenges and way-forward. Drug Discov Ther. 2021;15(1):42-3.

28. Mukwenha S, Dzinamarira T, Mugurungi O, Musuka G. Maintaining robust HIV and tuberculosis services in the COVID-19 era: A public health dilemma in Zimbabwe. Int J Infect Dis. 2020;100:3945.

29. World Health Organization. summary of the global HIV epidemic. Geneva; 2019. Available from: https://www.who.int/gho/hiv/en/.

30. Ooms G. COVID-19 and it's far-reaching health impacts in sub-Saharan Africa. Health Action International; 2020.

31. Corona Scanner. Realtime coronavirus statistics, Ethiopia. 2021. Available from: https://coronascanner.com/country/ethiopia.

32. Kibret GD, Ferede A, Leshargie CT, et al. Trends and spatial distributions of HIV prevalence in Ethiopia. Infect Dis Poverty. 2019;8:90. https://doi.org/10.1186/s40249-019-0594-9.

33. Swaminathan N, Moussa P, Mody N, Lo KB, Patarroyo-Aponte G. COVID-19 in HIV-infected patients: A case series and literature review. J Med Virol. 2021;93(5):2557-63.

34. Kowalska JD, Kase K, Vassilenko A, et al. The characteristics of HIV-positive patients with mild/asymptomatic and moderate/severe course of COVID-19 disease-A report from Central and Eastern Europe. Int J Infect Dis. 2021;104:293-6.

35. Calza L, Bon I, Tadolini M, et al. COVID-19 in patients with HIV-1 infection: a single-centre experience in northern Italy. Infection. 2021;49(2):333-7. 
36. Yang R, Gui X, Zhang Y, Xiong Y, Gao S, Ke H. Clinical characteristics of COVID-19 patients with HIV coinfection in Wuhan, China. Expert Rev Respir Med. 2021;15(3):403-9.

37. Ssentongo P, Heilbrunn ES, Ssentongo AE, et al. Epidemiology and outcomes of COVID-19 in HIVinfected individuals: a systematic review and meta-analysis. Sci Rep. 2021;11(1):6283. Published 2021 Mar 18.

38. Liang M, Luo N, Chen M, et al. Prevalence and Mortality due to COVID-19 in HIV Co-Infected Population: A Systematic Review and Meta-Analysis [published online ahead of print, 2021 May 3]. Infect Dis Ther. 2021;1-19.

39. Pizzirusso M, Carrion-Park C, Clark US, Gonzalez J, Byrd D, Morgello S. Physical and Mental Health Screening in a New York City HIV Cohort During the COVID-19 Pandemic: A Preliminary Report. J Acquir Immune Defic Syndr. 2021;86(3):e54-60.

40. Lee KW, Yap SF, Ngeow YF, Lye MS. COVID-19 in People Living with HIV: A Systematic Review and Meta-Analysis. Int J Environ Res Public Health. 2021;18(7):3554. Published 2021 Mar 30.

41. Thekkur P, Tweya H, Phiri S, et al. Assessing the Impact of COVID-19 on TB and HIV Programme Services in Selected Health Facilities in Lilongwe, Malawi: Operational Research in Real Time. Trop Med Infect Dis. 2021;6(2):81. Published 2021 May 19.

42. Abdela SG, Berhanu AB, Ferede LM, van Griensven J. Essential Healthcare Services in the Face of COVID-19 Prevention: Experiences from a Referral Hospital in Ethiopia. Am J Trop Med Hyg. 2020;103(3):1198-200.

43. Muwanguzi PA, Kutyabami $\mathrm{P}$, Osingada $\mathrm{CP}$, et al. Conducting an ongoing HIV clinical trial during the COVID-19 pandemic in Uganda: a qualitative study of research team and participants' experiences and lessons learnt. BMJ Open. 2021;11(4):e048825. Published 2021 Apr 21.

44. Kalichman SC, Shkembi B, Kalichman MO, Eaton LA. Trust in health information sources and its associations with COVID-19 disruptions to social relationships and health services among people living with HIV. BMC Public Health. 2021;21(1):817. Published 2021 Apr 28.

45. Jarolimova J, Yan J, Govere S, et al. Medical Mistrust and Stigma Associated with COVID-19 Among People Living with HIV in South Africa [published online ahead of print, 2021 May 17]. AIDS Behav. 2021;1-11.

46. Celestin K, Allorant A, Virgin M, et al. Short-Term Effects of the COVID-19 Pandemic on HIV Care Utilization, Service Delivery, and Continuity of HIV Antiretroviral Treatment (ART) in Haiti. AIDS Behav. 2021;25(5):1366-72.

47. Gwadz M, Campos S, Freeman R, et al. Black and Latino Persons Living with HIV Evidence Risk and Resilience in the Context of COVID-19: A Mixed-Methods Study of the Early Phase of the Pandemic. AIDS Behav. 2021;25(5):1340-60.

48. Muhula S, Opanga Y, Oramisi V, et al. Impact of the First Wave of the COVID-19 Pandemic on HIV/AIDS Programming in Kenya: Evidence from Kibera Informal Settlement and COVID-19 Hotspot Counties. Int J Environ Res Public Health. 2021;18(11):6009. Published 2021 Jun 3. 
49. Thekkur P, Takarinda KC, Timire C, et al. Operational Research to Assess the Real-Time Impact of COVID-19 on TB and HIV Services: The Experience and Response from Health Facilities in Harare, Zimbabwe. Trop Med Infect Dis. 2021;6(2):94. Published 2021 May 31.

50. Mbithi I, Thekkur P, Chakaya JM, et al. Assessing the Real-Time Impact of COVID-19 on TB and HIV Services: The Experience and Response from Selected Health Facilities in Nairobi, Kenya. Trop Med Infect Dis. 2021;6(2):74. Published 2021 May 10.

\section{Figures}

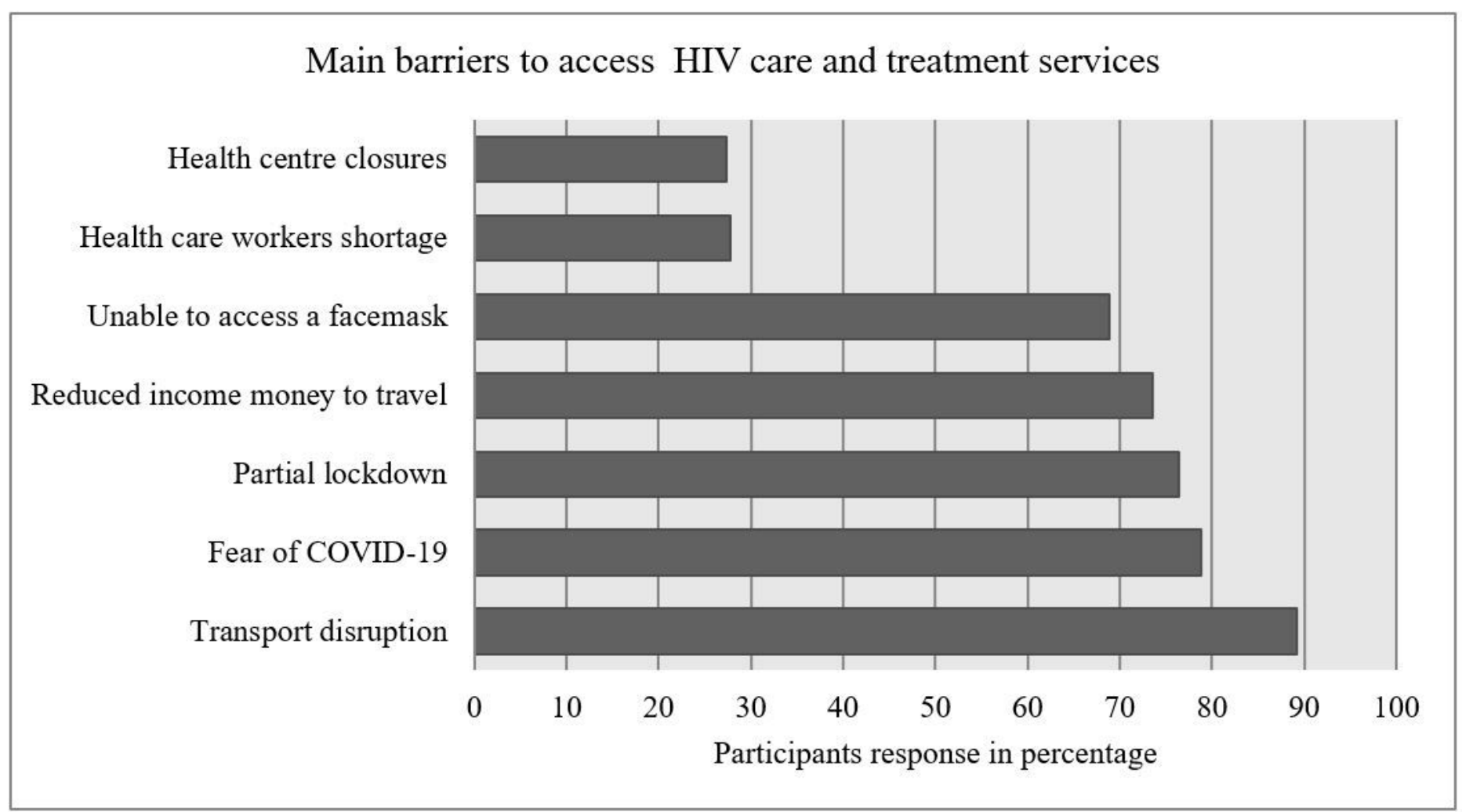

\section{Figure 1}

Response of study participants on barriers to access health care and treatment during the pandemic, Addis Ababa, Ethiopia May 2021. 